SELECCIONES MATEMÁTICAS
Universidad Nacional de Trujillo
ISSN: $2411-1783$ (Online)
Vol. $05(2): 193-203(2018)$

\title{
Simulación Numérica de Ondas Viajeras del Sistema FitzHugh-Nagumo
}

\section{Numerical Simulation of Traveling Waves of the FitzHugh-Nagumo System}

\author{
C. E. Rubio-Mercedes* and Glauce Barbosa Verão ${ }^{\dagger}$
}

Received, Nov. 14, 2018

Accepted, Dec. 22, 2018

DOI: http://dx.doi.org/10.17268/sel.mat.2018.02.06

\begin{abstract}
Resumen
El sistema FitzHugh-Nagumo tiene un tipo especial de solución llamada onda viajera, la cual tiene la forma $u(x, t)=\phi(x-\mu t)$ y $w(x, t)=\psi(x-\mu t)$, y es una solución estable en el tiempo. Nuestro interés es caracterizar numéricamente el perfil de una onda viajera $(\phi, \psi)$ y su velocidad de propagación $\mu(t)$. Con un cambio de variables, transformamos el problema de encontrar las soluciones en coordenadas originales a un problema de encontrar los equilibrios en un nuevo sistema de coordenadas llamado coordenadas móviles o sistema de coordenadas no locales. Con ejemplos numéricos demostraremos que las soluciones del sistema de EDPs en coordenadas no locales converge a una onda viajera del problema original. El sistema de coordenadas no locales también permite calcular la velocidad de propagación en forma exacta.

Palabras clave. Onda viajera, Solución estable, EDPs, Coordenadas móviles.
\end{abstract}

\begin{abstract}
The FitzHugh-Nagumo system has a special type of solution called traveling wave, which has the form $u(x, t)=\phi(x-\mu t)$ and $w(x, t)=\psi(x-\mu t)$, which is a stable solution over time. Our interest is to numerically characterize the profile of a traveling wave $(\phi, \psi)$ and its propagation speed $\mu(t)$. With a change of variables, we transform the problem of finding the solutions in original coordinates to a problem of finding the equilibria in a new coordinate system called mobile coordinates or non-local coordinate system. With numerical examples we will demonstrate that the solutions of the system of EDPs in non-local coordinates converge to a traveling wave of the original problem. The non-local coordinate system also allows to calculate the exact propagation speed.
\end{abstract}

Keywords. Traveling wave, Stable solution, PDEs, Mobile coordinates.

1. Introducción. Procesos biológicos cómo el ciclo de crecimiento y división celular, fluctuaciones de tensión en membranas excitables, son ejemplos importantes de ritmos complejos en los seres vivos, los cuales no son constantes en el tiempo. El interés en estas fluctuaciones ha sido estimulado por conocimientos de que sistemas dinámicos determinísticos pueden presentar dinámica caotica en el tiempo: ritmos no periódicos sensibles a las condiciones iniciales. Los seres vivos producen señales biológias, que pueden ser eléctricas, mecánicas o químicas. Estas señales son de interés para el diagnóstico, observación de enfermos o simplemente para investigación biomédica. Para estudiar la presencia de ondas viajeras y caos en sistemas biológicos, como los pulsos neuronales en el cerebro y latidos cardiacos en el corazón, son necesarios modelos teóricos que generen señales coherentes con los reales, además de datos experimentales, en condiciones controladas. En este trabajo simulamos computacionalmente las soluciones llamadas ondas viajeras de las ecuaciones dinámicas de tipo reacción-difusión presentes en la vibración de pulsos neuronales en el cerebro y la presencia de ondas reentrantes en el corazón. Las simulaciones numéricas

* UEMS-Universidade Estadual de Mato Grosso do Sul, Dourados, MS, Brasil (cosme@uems.br ).

†USP-Universidade de São Paulo, São Paulo, Brasil (glaucematematica@gmail.com), 
sirven para analizar visual e interactivamente el comportamiento cualitativo y cuantitativo de los sistemas en estudio, modelados por ecuaciones diferenciales parciales (PDEs).

Este trabajo es organizado de la siguiente manera. En la Sección 2 presentamos el modelo de estudio en coordenadas locales. En la Sección 3 se establece el modelo en coordenadas no locales. Los resultados numéricos son discutidos en la Sección 4 y finalmente las conclusiones seran dadas en la Sección 5.

2. El Modelo. Las características más sobresalientes del potencial de acción y su dinámica, fueron modeladas por Hodgkin y Huxley con un sistema de cuatro ecuaciones en 1952 [1]. Todos los elementos involucrados, tienen un significado biofísico, sin embargo, la complejidad matemática dificulta demasiado su análisis.

Con el afán de comprender la esencia dinámica del fenómeno de excitabilidad, FitzHugh en 1961 construyó un sistema de dos ecuaciones basado en la ecuación de Van der Pol [2]. Simultáneamente a FitzHugh, Jin-ichi Nagumo propuso, en 1962, un circuito eléctrico no lineal, gobernado por un sistema de dos ecuaciones también semejantes a los de Van Der Pol [3]. Actualmente, el modelos simplificado propuesto por estos autores, se le conoce como sistema de FitzHugh-Nagumo (FHN). Un término difusivo fue incorporado a la primera ecuación del sistema FHN por Panfilov [4] en 1984, con el intento de representar mejor el periodo refractario importante en el estudio de arritmias reentrantes. Hoy, la importancia de este modelo trasciende el ámbito de la Biofísica y la Neurofisiología, siendo de interés para los profesionales de otras ramas de la ciencia que necesitan comprender la constelación de fenómenos no lineales, que son concomitantes al fenómeno de excitabilidad. Particularmente el modelo es de interés para ingenieros y científicos interesados en el estudio de sistemas excitables.

En general, métodos numéricos son usados para obtener una caracterización del perfil y velocidad de propagación $c$. Para esto se fija un domínio comoputacional finito. Sin embargo, el comportamiento de la solución en un domínio finito pude ser completamente diferente que cuando se considera el domínio completo. Para resolver este problema, usamos el método de coordenadas no locales (o coordenadas móviles) que consiste en una transformación de coordendas que presentaremos en la siguiente sección.

Consideremos el sistema FHN,

$$
\left\{\begin{array}{l}
u_{t}=u_{x x}+f(u)-w, \\
w_{t}=\varepsilon(u-\gamma w),
\end{array} \quad x \in R, \quad t>0 .\right.
$$

donde $\varepsilon>0, \gamma>0$ son parametros reales y $f$ es una función no lineal cubica relacionada a la corriente iónica [3], dada por $f(u)=u(1-u)(1-a)$. Es decir, $f$ es de clase $C^{2}$ que satisface las siguiente condiciones:

(H1) Existe $a, 0<a<1$ tal que $f(0)=f(a)=f(1)=0, \quad f^{\prime}(0)<0, \quad f^{\prime}(1)<0$ y $f^{\prime}(a)>0$;

(H2) $f(u)>0$ para $u<0$ o $a<u<1$ y $f(u)<0$ para $0<u<a$ o $u>1$;

(H3) $\int_{0}^{1} f(s) d s>0$.

Ondas viajeras son sluciones de (2.1) las cuales tienen la forma

$$
u(x, t)=\phi(\xi), \quad w(x, t)=\psi(\xi), \quad \xi=x-c t
$$

donde $(\phi, \psi)$ es su perfil de la onda viajera y $c>0$ es su velocidad de propagación. Naturalmente, si $\phi$ y $\psi$ son funciones reales de clase $C^{2}$, entonces la terna $(\phi, \psi, c)$ es solución del sistema

$$
\left\{\begin{aligned}
\phi^{\prime \prime}-c \phi^{\prime}+f(\phi)-\psi & =0 \\
-c \psi^{\prime}+\varepsilon(\phi-\gamma \psi) & =0 .
\end{aligned}\right.
$$

Para provar la existencia de ondas viajeras, es conveniente transformar (2.3) en un sistema de primer orden que depende del parametro $c$ dado por

$$
\left\{\begin{aligned}
u^{\prime}(\xi) & =v(\xi) \\
v^{\prime}(\xi) & =c v(\xi)-f(u(\xi))+w(\xi) \\
w^{\prime}(\xi) & =\frac{\varepsilon}{c}(u(\xi)-\gamma w(\xi)) .
\end{aligned}\right.
$$

El sistema anterior puede ser escrito en forma vectorial como

$$
V_{x}=F(V), \quad V=(u, v, w)^{T}, \quad \lim _{x \rightarrow \pm \infty} V(x)=V_{ \pm}=\left(u_{ \pm}, 0, w_{ \pm}\right)
$$

El número de puntos de equilibrio de (2.5) depende solamente del valor de $\gamma$. Por lo tanto, los pulsos viajeros y frentes viajeras corresponden, respectivamente, a órbitas homoclínicas y heteroclínicas 
que conectan dos estados estacionarios $V_{-}$a $V_{+}$del sistema dinámico (2.5) para un valor específico del parámetro $c$. Para pequeños valores de $\gamma,(2.5)$ tiene un único punto de equilibrio de manera que el sistema presenta soluciones de tipo pulsos viajeros y para $\gamma$ suficientemente grande, mayor que $1 /(2-a)(1-2 a)$, (2.5) presenta 3 puntos de equilibrio y existe la propagación de frentes viajeras [9]-[13].

Supomgamos que $\gamma$ satisface la siguiente hipotesis:

(H4) $\gamma>0$ suficientemente grande para que $f(u)=\frac{1}{\gamma} u$ tenga tres raíces reales $0<a<u_{1}<u_{2}<1$ y

$$
\int_{0}^{u_{2}}\left(f(u)-\frac{1}{\gamma} u\right) d u>0
$$

Con las hipótesis (H1)-(H4), el sistema (2.4) tiene tres puntos de equilibrio $p_{0}=(0,0,0), p_{1}=$ $\left(u_{1}, 0, \frac{1}{\gamma} u_{1}\right)$ y $p_{2}=\left(u_{2}, 0, \frac{1}{\gamma} u_{2}\right)$. Nuestro interes inicial es estudiar la clase de ondas viajeras llamdas de frentes viajares que conectan los puntos $p_{0}$ y $p_{2}$, esto es, estudiar la existencia, estabilidad y aproximación de ondas viajeras $(\phi, \psi)$ del sistema (2.1) que satisfaga

$$
\left(\phi(-\infty), \phi^{\prime}(-\infty), \psi(-\infty)\right)=(0,0,0) \quad \mathrm{y} \quad\left(\phi(+\infty), \phi^{\prime}(+\infty), \psi(+\infty)\right)=\left(u_{2}, 0, \frac{u_{2}}{\gamma}\right)
$$

Existencia y estabilidad de este tipo de soluciones puede encontrarse en [5], [6] y [13].

3. Sistema No Local. El método de coordenadas no locales (o método de coordenadas móviles) consiste en el cambio de variables: $u(x, t)=p(x-\eta(t), t)$ y $w(x, t)=q(x-\eta(t), t)$ y aproximando el perfil $(\phi, \psi)$ por equilibrios de la ecuación no local

$$
\left\{\begin{array}{l}
p_{t}=p_{x x}-\eta^{\prime}(t) p_{x}+f(p)-q \\
q_{t}=-\eta^{\prime}(t) q_{x}+\varepsilon(p-\gamma q)
\end{array}\right.
$$

Con las nuevas variables, necesitamos una condición de fase, es decir, una ecuación para $\eta(t)$, para que el problema (3.1) este bien definido.

La elección para $\eta(t)$ se basa en que el término no local $\eta^{\prime}(t) \rightarrow c$ cuando $t \rightarrow \infty$, donde $c$ es la velocidad de propagación la onda viajera, soluciones del sistema (2.1).

Multiplicando por $\phi^{\prime}$ la primera ecuación del sistema (2.3) e integrando en $R$, usando el hecho que $\phi^{\prime}(\infty)=0$, tenemos

$$
c=-\frac{\left\langle f(\phi)-\psi, \phi^{\prime}\right\rangle}{\left\langle\phi^{\prime}, \phi^{\prime}\right\rangle}=-\frac{\left\langle f(\phi), \phi^{\prime}\right\rangle-\left\langle\psi, \phi^{\prime}\right\rangle}{\left\langle\phi^{\prime}, \phi^{\prime}\right\rangle}
$$

donde $\langle\cdot, \cdot\rangle$ es el producto interno en $L^{2}(R)$.

La ecuación equivalente para el sistema (3.1) es dada por

$$
\mu(t):=\eta^{\prime}(t)=\frac{A-\left\langle q(\cdot, t), p_{x}(\cdot, t)\right\rangle}{\left\langle p_{x}(\cdot, t), p_{x}(\cdot, t)\right\rangle}
$$

o, equivalentemente

$$
\eta(t)=\int_{0}^{t} \frac{A-\left\langle q(\cdot, s), p_{x}(\cdot, s)\right\rangle}{\left\langle p_{x}(\cdot, s), p_{x}(\cdot, s)\right\rangle} d s
$$

donde $A=\int_{0}^{u_{2}} f(s) d s$ para frentes viajeras.

El objetivo de este estudio es poder aproximar numéricamente el perfil y la velocidad de la onda viajera a través de equilibrios del sistema (3.1). Para usar métodos numéricos es necesarios truncar el dominio espacial y por eso, en la Sección 4 analizamos el problema no local en un intervalo finito con ciertas condiciones de contorno (CC), esto es:

$$
\left\{\begin{array}{l}
p_{t}=p_{x x}-\frac{A-\left\langle q, p_{x}\right\rangle}{\left\langle p_{x}, p_{x}\right\rangle} p_{x}+f(p)-q, \\
q_{t}=-\frac{A-\left\langle q, p_{x}\right\rangle}{\left\langle p_{x}, p_{x}\right\rangle} q_{x}+\varepsilon(p-\gamma q), \\
p(x, 0)=u_{0}(x) \text { and } q(x, 0)=w_{0}(x) .
\end{array}\right.
$$


Las CCs del sistema anterior deben ser escojidas de forma a satisfacer la onda viajara del problema en estudio, ya sea para la obtención de frentes viajeras o pulsos viajeros. $[-J, J]$

Para resolver numericamente el sistema no local usaremos el siguiente sistema en un intervalo finito

$$
\begin{aligned}
& u_{t}=u_{x x}+\mu u_{x}+f(u)-w \\
& w_{t}=\mu w_{x}+\epsilon(u-\gamma w) \text { para } \quad-J \leq x \leq J \\
& \mu\left\langle u_{x}, u_{x}\right\rangle+A-\left\langle w, u_{x}\right\rangle=0 \\
& u(x, 0)=u_{0}(x) \text { y } w(x, 0)=w_{0}(x) \\
& u(-J, t)=0 \text { y } w(-J, t)=0 \text { para } t>0 .
\end{aligned}
$$

Condiciones de contorno de tipo Dirichlet (CCD) son usadas para simular frentes viajeras. Tambien se pueden usar condiciones de contorno de Neumann homogéneas (CCNH) debido a que se espera que en los límites del dominio tengamos $u_{x}=0$.

4. Resultados Numéricos. En esta sección consideramos algunos ejemplos de simulación de las ondas viajeras de tipo frentes y pulsos. Usaremos el Software de distribución libre FreeFem $++[7]$,[8], el cual es un ambiente de desarrollo integrado de alto nivel para resolver numéricamente EDPs en dominios 2D y 3D. Además de ser una herramienta ideal para la enseñanza del método del elemento finito (MEF), es perfecto para la investigación y examinar nuevas aplicaciones complejas en problemas variados de física. FreeFem ++ tiene un generador de malla automático avanzado, capaz de usar mallas adaptativas; tiene un potente solucionador de sistemas matriciales lineares. También tiene varios elementos finitos triangulares y tetraedros que incluyen elementos discontinuos.

En el esquema numérico, para la propagación en la coordenada temporal, usamos un esquema semi implícito, el cual aproxima la dervida termporal con una diferencia progresiva entre los pasos de evolución $n$ y $n+1$, el termino difusivo es aproximado en el paso $n+1$ y para el termino no lineal es usado el valor conocido en el paso anterior $n$.

4.1. Simulación de Frentes Viajeras. Consideramos los parámetros $\epsilon=0.01, a=0.25$ y $\gamma=20$, con condiciones iniciales $(\mathrm{CI})$ :

$$
u_{0}(x)=(x+J) / 2 J, \quad w_{0}(x)=(x+J) / 40 J
$$

Para tales valores de parametros, $\mathrm{F}$ dada en (2.5) tiene tres ceros de modo que existe propagación de frentes viajeras. Dividimos el dominio espacial en 400 elementos cuadráticos y el paso de propagación temporal $\Delta t=0.5$. Las Figs. 4.1(a-b) muestran los gráficos de la variación espacio-temporal de la componente $u$, del sistema (2.1) en Fig. 4.1(a) y en coordenadas no locales, sistema (3.6), en Fig. 4.1(b). En la Fig. 4.1(c) presentamos la variación espacio-temporal de la componente $w$, del sistema (3.6), y en la Fig.4.1 (d) el valor de la velocidad de propagación $\mu(t)$, la cual converge para $\mu(t)=-0.247255$.

En la Fig. 4.2(a) calculamos la norma del error entre iteraciones sucesivas para las componentes $u$ (línea roja) y $w$ (línea azul) del sistema FHN en coordenadas no locales. En la Fig. 4.2(b) presentamos la norma del error entre iteraciones sucesivas para varios valores del números de elementos en la división espacial para la componente $u$. Esas curvas estan en escala logarítmica y los resultados demuestran claramente que hay convergencia para una frente viajera de velocidad constante.

Como un segundo ejemplo de propagación de frentes viajeras consideramos una CI:

$$
u_{0}(x)=(x+J)(1-\cos (x / 2)) / 2 J(1-\cos (J / 2)), \quad w_{0}(x)=0
$$

Los resultados de este ejemplo de simulación son presentados en la Fig. 4.3, donde en Fig.4.3(a) presentamos la distribución espacio-temporal de la componente $u$, mientras que la Fig.4.3(b) es la componente $w$, ambas en coordenadas no locales en 4.3(c) presentamos la velocidad de propagación $\mu(t)$. En este ejemplo, podemos observar que el valor de la velocidad de propagación también converge para $\mu(t)=-0.247255$ como en el primer ejemplo de esta sección.

En la Fig. 4.4(a) calculamos la norma del error entre iteraciones sucesivas para las componentes $u$ (línea roja) y $w$ (línea azul) del sistema FHN en coordenadas no locales y CI dadas en (4.2). En la 


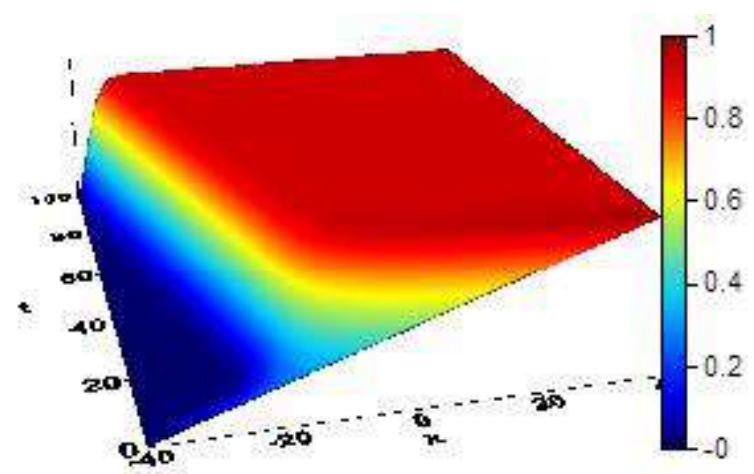

(a)

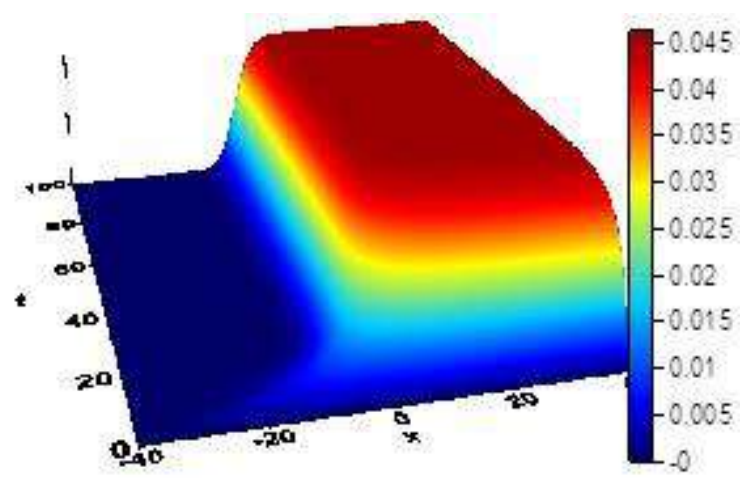

(c)

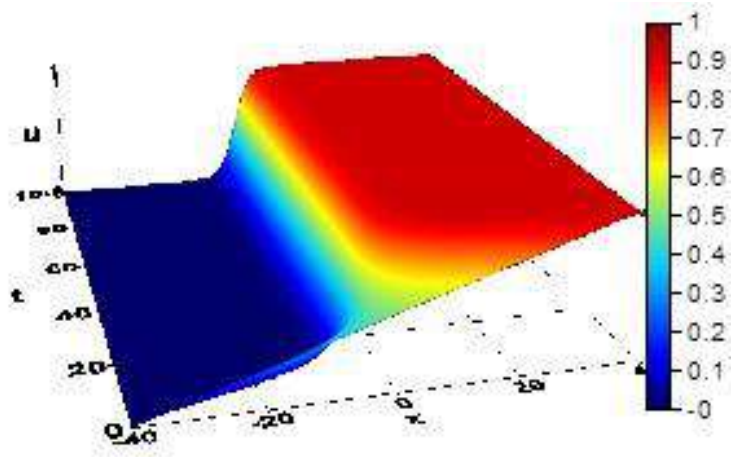

(b)

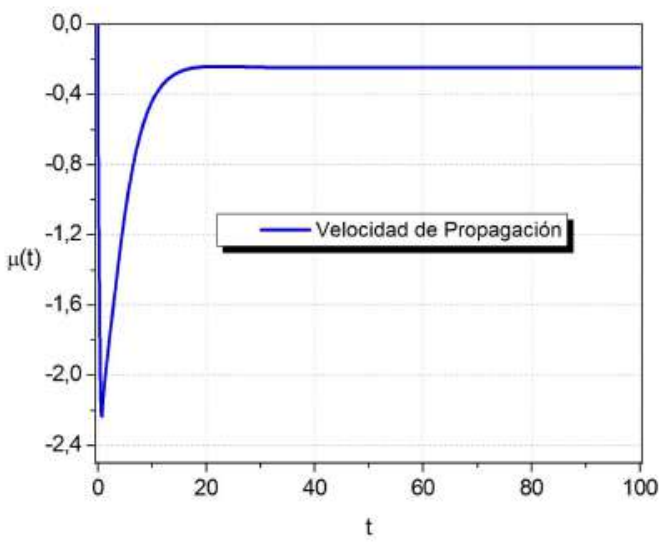

(d)

Figura 4.1. Perfil espacio-temporal de la frente viajera, componente $u$, para el sistema FHN, sistema(2.1), en coordenadas locales (a), perfil de la frente viajera en el sistema FHN, sistema (3.6), en coordenadas no locales para la componente $u$ (b), componente $w$ para el sistema FHN en coordenadas no locales, sistema (3.1)(c) y velocidad de propagación $\mu$ (d).

Fig. 4.4(b) presentamos la norma del error entre iteraciones sucesivas para varios valores del números de elementos en la división espacial para la componente $u$. Estos resltados estan estrictamente de acuerdo con las simulaciones de [10] de la cual fueron adquiridas.

4.2. Simulación de Pulsos Viajeros. Pulsos viajeros del sistema FHN son obtenidos con valores de parámetros: $\epsilon=0.0035, \alpha=0.13$ y $\gamma=2.7$. Para estos valores la función $F(u, v, w)$, dada en (2.5), admite un único cero $(u, v, w)=(0,0,0)$ y se obtienen pulsos viajeros en la propagación. Como CI consideramos la función rampa para la componente $u$ y la componente $w$ con valor igual a 0 , dadas por

$$
u_{0}(x)=(x+|x|) / 2, \quad w_{0}(x)=0
$$

Con CC de Dirichlet homogeneas (CCDH), el pulso viaja para el lado izquierdo como esperando cuando usamos el sistema FHN en coordenadas locales dado en (2.1), ver Fig. 4.5(a) para la componente $u$ y Fig. 4.5(b) para la componente $w$. De la misma forma como los ejemplos de la simulación de frentes viajeras presentados en la Sección 4.1, el método aplicado en coordenadas no locales captura el perfil del pulso en el centro de dominio, como puede ser visto en la Fig. 4.5(c) para la componente $u$ y Fig.4.5(d) para $w$. Con el método en coordenadas no locales, se obtiene la velocidad de propagación del pulso, la cual converge para $\mu(t)=-0.487484$. Este valor es obtenido con CCDH en la segunda ecuación del sistema dado en (3.6). Con CCNH para la segunda ecuación del sistema FHN (3.6), no hemos tenido resultados satisfactorios de propagación de pulsos, este es un problema en abierto y necesitamos hacer más experimentos de validación de forma a descubrir la razón de esta dificultad. 


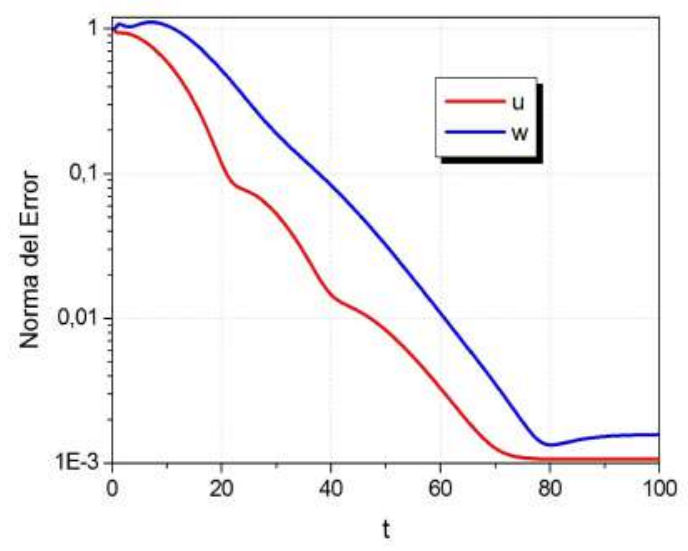

(a)

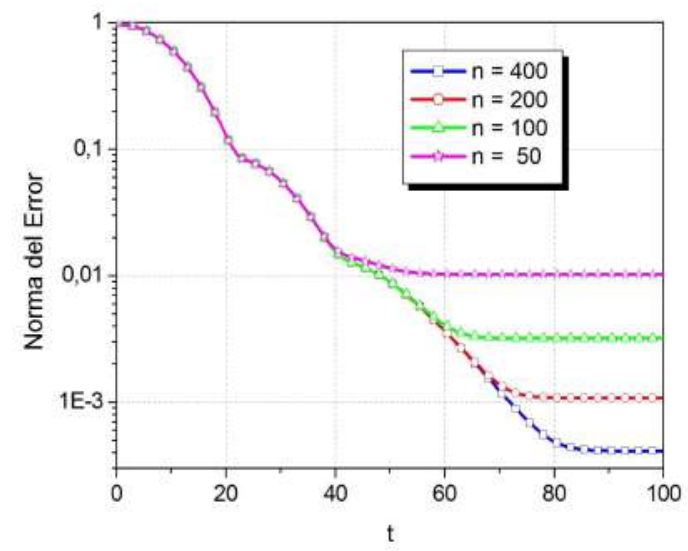

(b)

FiguRA 4.2. Norma del error entre iteraciones sucesivas para las componentes $u$ y $w$ en (a) y norma del error entre iteraciones sucesivas para varios números de elementos en la coordenada espacial para la componente $u$, ambos para la CI dada en (4.1) y con el sistema de coordenadas no locales (3.6)

Debido a las dificultades encontradas al simular pulsos viajeros con el sistema en coordenadas no locales (3.6) y CCNH, optamos por usar un dominio computacional suficientemente grande de tal forma que el pulso no llegue a los limites del dominio y así podamos usar CCDH.

Para este ejemplo de simulación de pulsos viajeros también calculamos la norma del error entre iteraciones sucesivas para la componente $u$ y $w$ del sistema (3.1), los resultados son presentados en la Fig.4.6(a). En la Fig.4.6(b) presentamos la norma del error para la componente $u$ en función del número de elementos del domínio computacional espacial.

4.3. Simulación de Bifurcación de Pulsos Viajeros. Un fenómeno interesante ocurre cuando el sistema es estimulado por una CI de tipo pulso cuadrado, donde $u_{0}(x)=0$ en todo el domínio computacional, excepto en el intervalo $[-5.5,5.5]$ en donde $u_{0}(x)=1$, ver Fig. Fig.4.7(a). Se generan dos pulsos viajeros, uno viajando para el lado izquierda y el otro viajando para el lado derecho del dominio, ver Fig. Fig.4.7(b). Responder cuál de los dos pulsos es capturado por el sistema de coordenadas no locales, es difícil y depende, por ejemplo, del método numérico usado y la forma de cálculo de $\mu(t)$. Experimentos numéricos nos muestran en este caso que, el método de coordenadas no locales falla en el cálculo de $\mu(t)$, dando un resultado prácticamente cero. Es necesario hacer las ssimulaciones con dos sistemas de ecuaciones [10]. Si en nuestro esquema numérico consideramos la velocidad fija en $\mu(t)=-0.487484$, capturamos el pulso que viaja al lado izquierdo mientras que el pulso derecho viaja hacia la frontera, ver Fig.4.7(c). Cuando fijamos la velocidad en $\mu(t)=0.487484$, capturado el pulso del lado derecho mientras el pulso del lado izquierdo viaja hacia la frontera, ver Fig. Fig.4.7(d). Este valor de la velocidad fue calculado por el método de coordenadas no locales y CI de tipo función rampa. Para varios pulsos viajeros, como es el caso de este ejemplo, se debe usar esquemas numéricos aplicados a varios sistemas de ecuaciones, el cual va depender de la cantidad de pulsos que queremos simular y calcular la velocidad de propagación en cada sistema [10].

Para determinar la potencia critica para tener bifurcación de pulsos, hacemos otro experimento numérico usando el sistema en coordenadas locales y verificaremos el efecto de la variación de la amplitud de la CI $u_{0}(x)$ del sistema FHN. Consideramos el parametro que define el dominio espacial con valor $J=100$ y tiempo final $T=200$, dividiendo el dominio espacial en 800 elementos cuadráticos y el paso de propagación $\Delta t=0.5$. Controlamos la intensidad de las CIs cambiando la amplitud de $u_{0}(x)$. Consideremos una CI de forma de pulso sinoidal, ver Fig.4.8(a), con amplitud igual a 2 en el intervalo $[-5.5,5.5]$, y de valor igual a 0 en el resto del dominio. Observamos que esa CI tiene un forma de la función seno y genera pulsos viajeros, tanto para el potencial $u$ como para la variable lenta $w$, que son simétricas con respecto a la ubicación central de la CI. Como se ilustra en las Figs.4.8(b-c), ambas ondas viajan con velocidades constantes com valores absoluto de aproximadamente $\mu(t)=0.49$ [10]. Del mismo modo que el ejemplo anterior, de CI de tipo rampa, cuando fijamos la velocidad en -0.49(0.49) el sistema de coordenadas no locales captura la onda viajera del lado izquierdo (derecho) siendo que la onda del lado derecho (izquierdo) se desplaza para la frontera del dominio. 


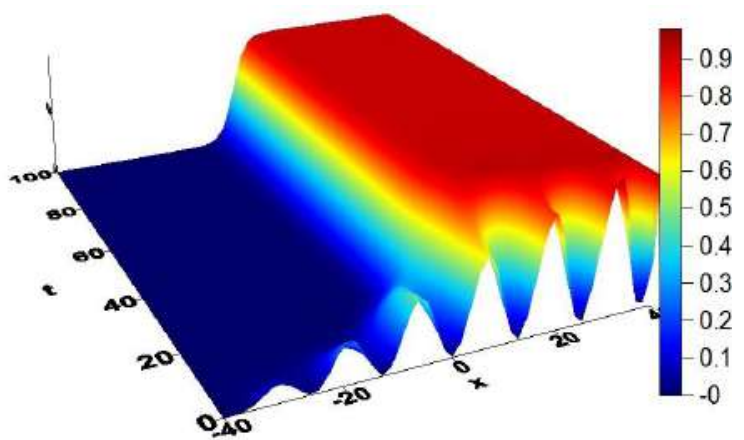

(a)

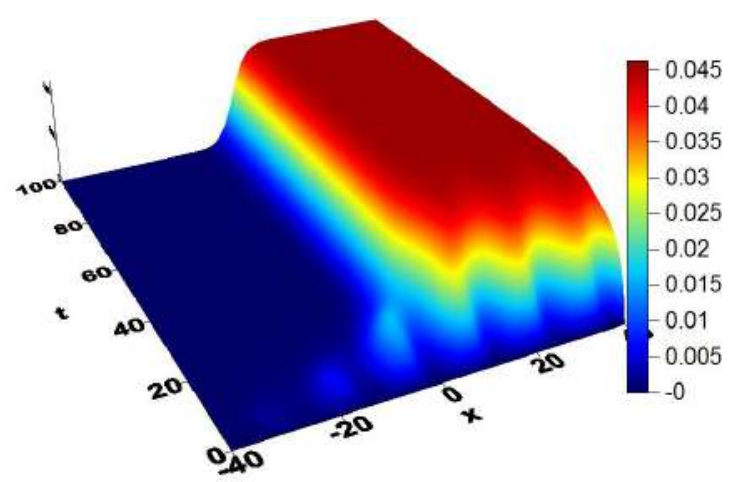

(b)

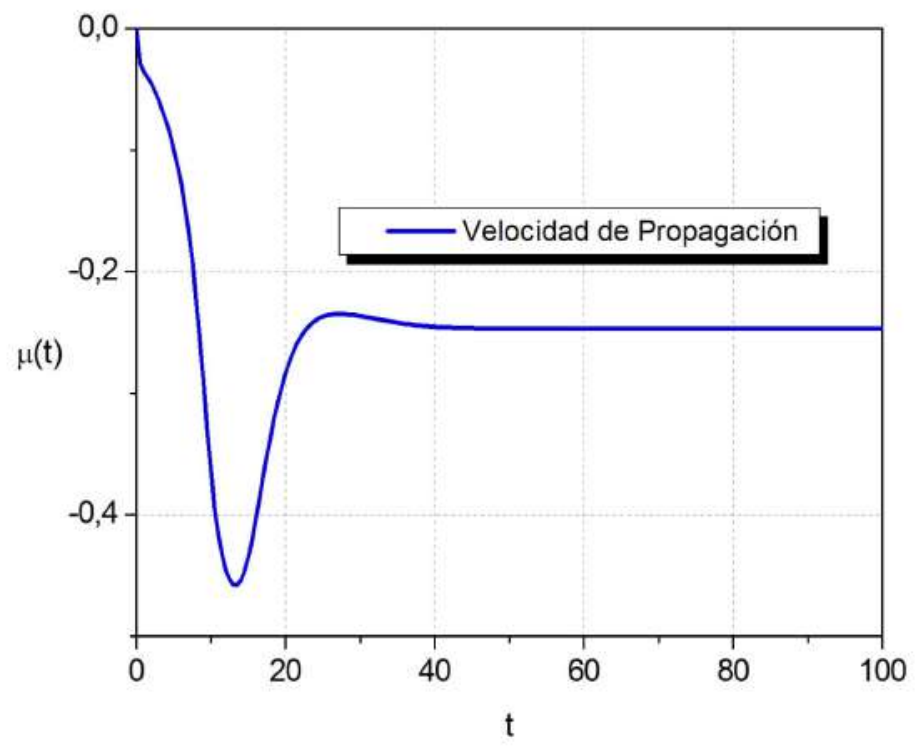

(c)

FiguRa 4.3. Solución espacio-temporal del sistema FHN en coordenadas no locales dado en (3.6) y CI dada en (4.2); componentes $u$ en (a), $w$ en (b) y velocidad de propagación $\mu(t)$ en (c).

Observamos que cuando la amplitud es igual 1, el sistema FHN todavía es estimulado por la CI, que se propagará de una manera similar a la presentada en las Figs. 4.8(b-c). En la Fig.4.9, simulamos la propagación de las soluciones del sistema $\mathrm{FHN}(2.1)$ cuando estimulamos con un pulso de forma como presentado en la ver Fig.4.8(a), de amplitud 0.5. Observamos que, en este caso, no se proporciona energía suficiente para excitar una onda viajera. La variable $w$ es dominante en el proceso, lo que hace que el potencial $u$ se aproxime a 0 rápidamente.

La potencia de los estimulos iniciales también está influenciada por el soporte de $u_{0}(x)$. Considerando una CI de amplitud 2 con soporte $[-2.5,2.5]$, observamos resultados similares a los resultados como obtenidos en la Fig. 4.9. El sistema FHN tampoco presenta soluciones de ondas viajeras en este caso. La existencia de una potencia crítica para emitir ondas viajeras de tipo pulsos con el sistema FHN se deduce de los resultados numéricos. Además, dadas ciertas restricciones para los estímulos, las condiciones del valor crítico pueden especificarse utilizando métodos numéricos.

Finalmente, presentamos un ejemplos de simulación de múltiples pulsos con el sistema FHN(2.1). Las condiciones para generar múltiples pulsos con el modelo de FHN se han estudiado en la literatura (véase, por ejemplo, [9]-[11]). Notemos que estos resultados teóricos son en su mayoría condiciones suficientes para soluciones de múltiples pulsos, que no excluyen otros patrones de soluciones. En esta simulación, consideramos el dominio computacional $[-400,400] \times[0,400]$ con $u_{0}(x)=1$ para $x \in[-100,-90] \cup[90,100]$ 


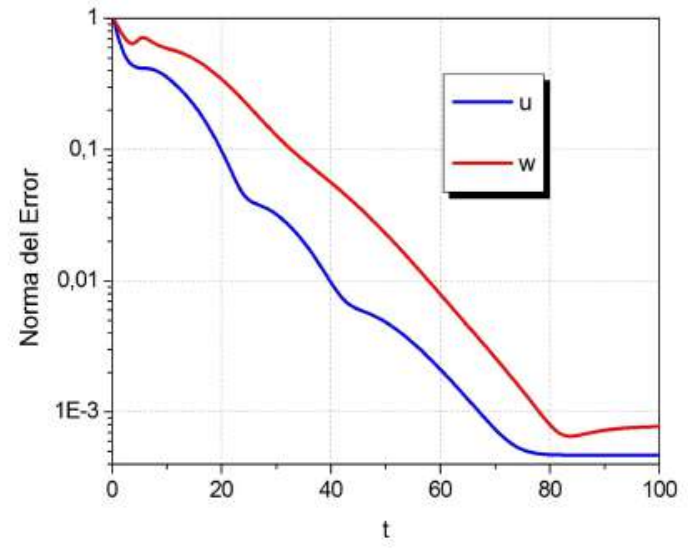

(a)

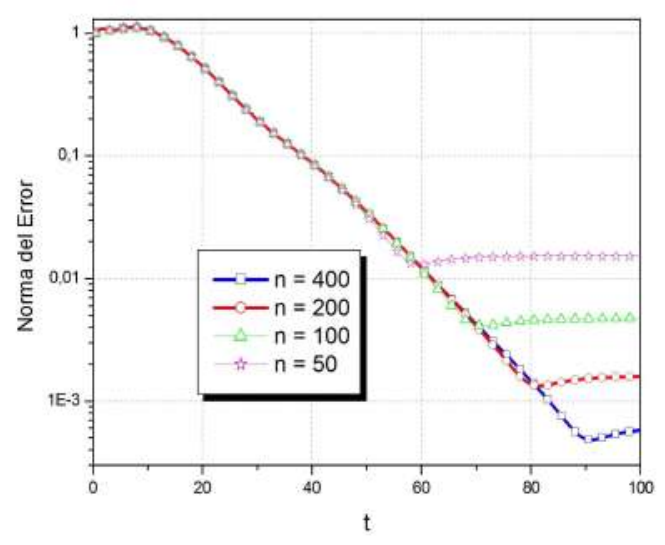

(b)

FiguRA 4.4. Norma del error entre iteraciones sucesivas para la componente u (línea azul) y componente $w$ (línea roja) (a). Norma del error entre iteraciones sucesivas para varios valores del número de elementos en la coordenada espacial (b) para el ejemplo de la CI dada em (4.2) y el sistema de coordenadas no locales dado en (3.6).

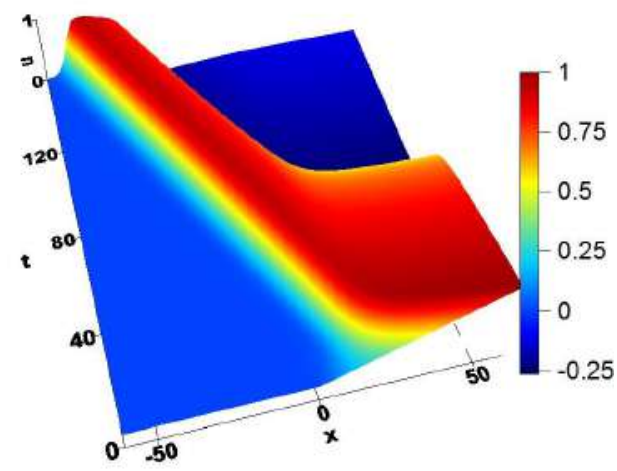

(a)

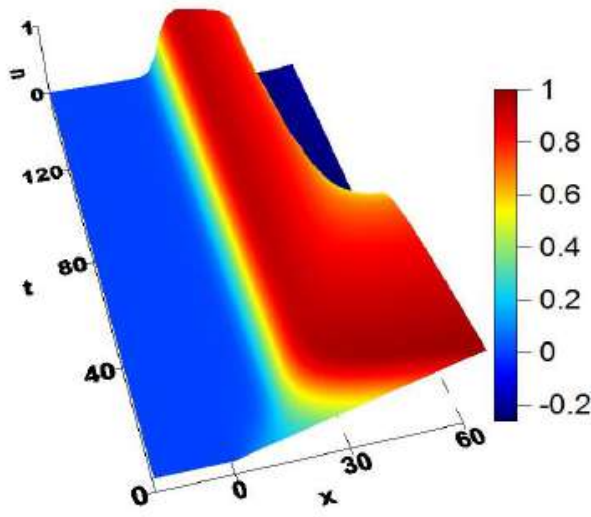

(c)

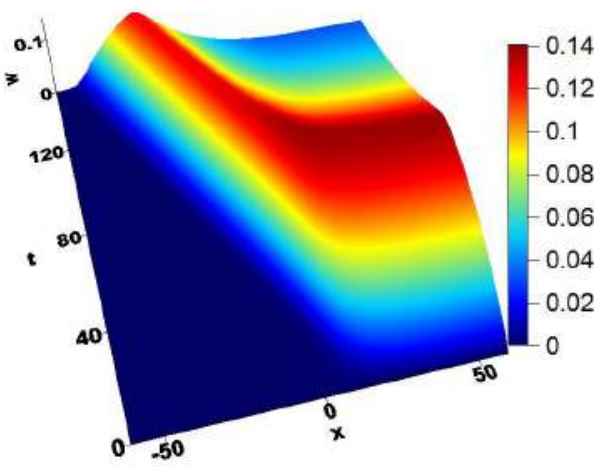

(b)

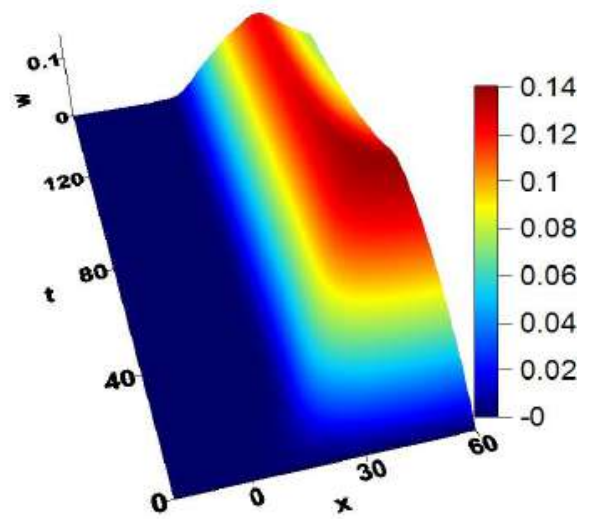

(d)

Figura 4.5. Simulaciones de pulsos viajeros con el sistema de coordenadas locales (2.1), componente u (a) y componente $w(b)$. Simulaciones de pulsos con el método en coordenadas no locales(3.6), componente u (c), componente $w$ (d). 


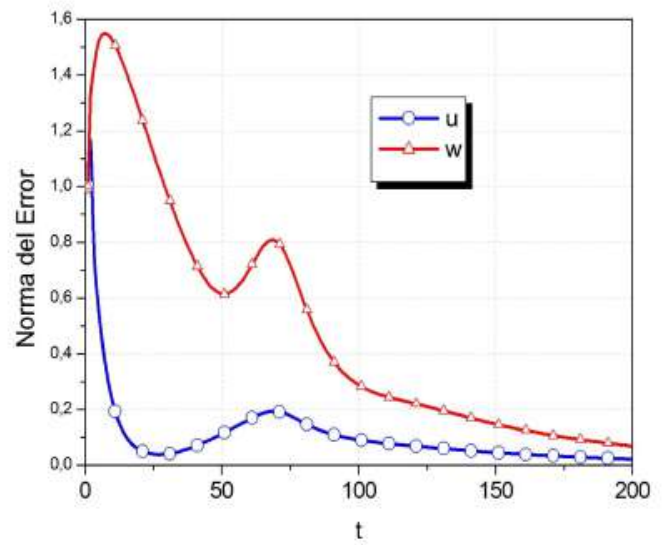

(a)

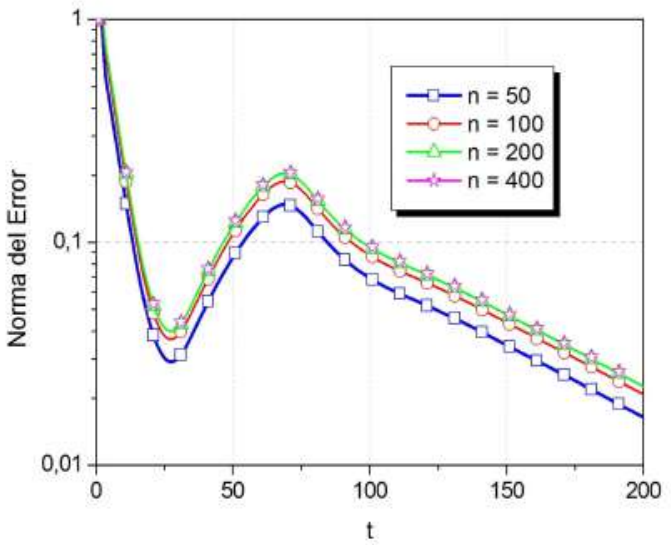

(b)

FiguRa 4.6. Norma del error entre iteraciones sucesivas para la componente u (línea azul) y componente $w$ (línea roja) (a) para la simulación de pulsos viajeros. Norma del error entre iteraciones sucesivas para la componente u para varios valores del número de elementos en la coordenada espacial (b). Ambos gráficos obtenidos con la CI dada en (4.3) y sistema en coordenadas no locales.

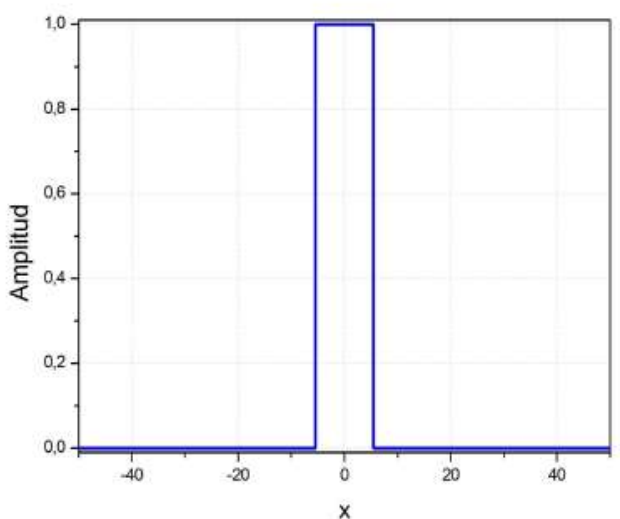

(a)

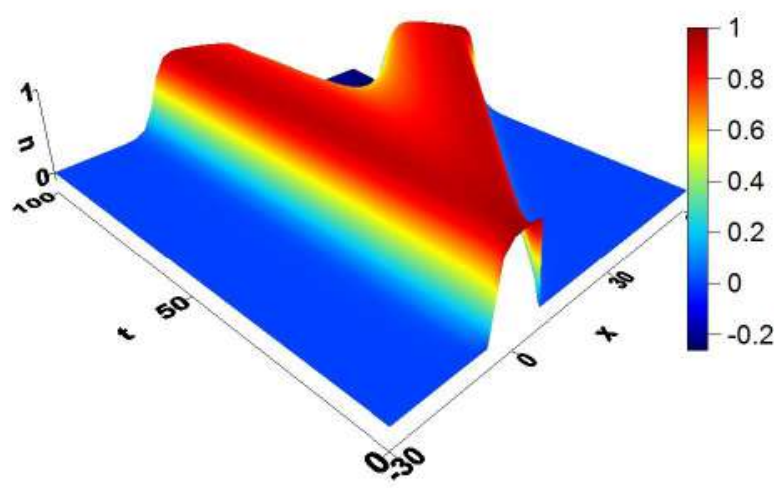

(c)

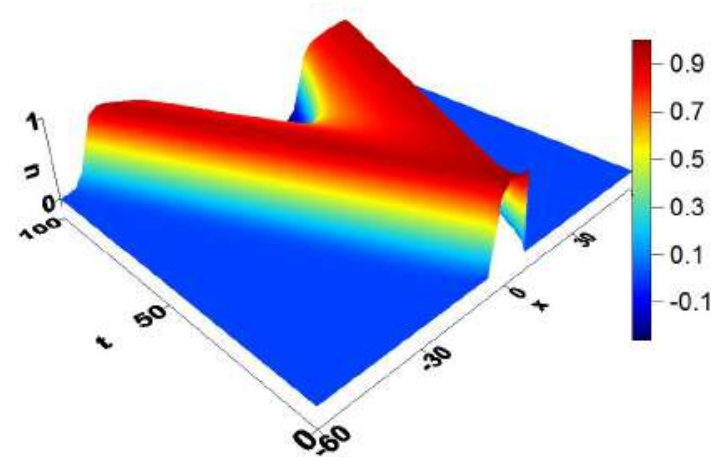

(b)

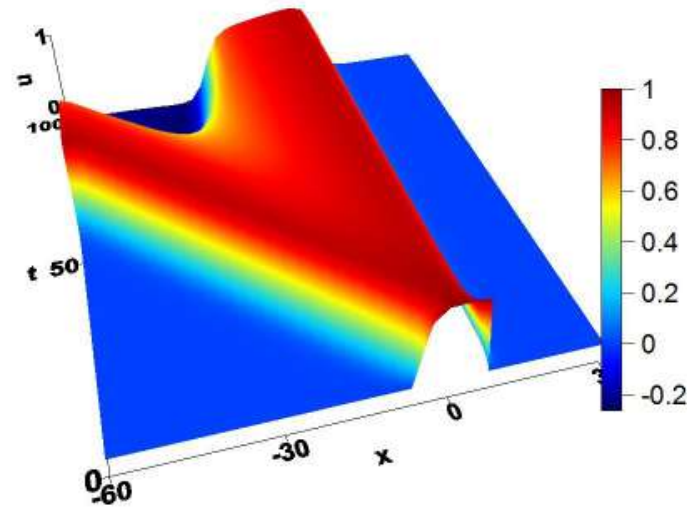

(d)

Figura 4.7. Simulaciones de fiburcación de pulsos para una CI de tipo pulso cuadrado: CI (a), componente u con el sistema de cordenadas locales (b), componente u con velocidad fija $\mu(t)=-0.487484$ (c) y componente $u$ con velocidad fija $\mu(t)=0.487484$ ambas con el sistema de coordenadas no locales $(d)$. 


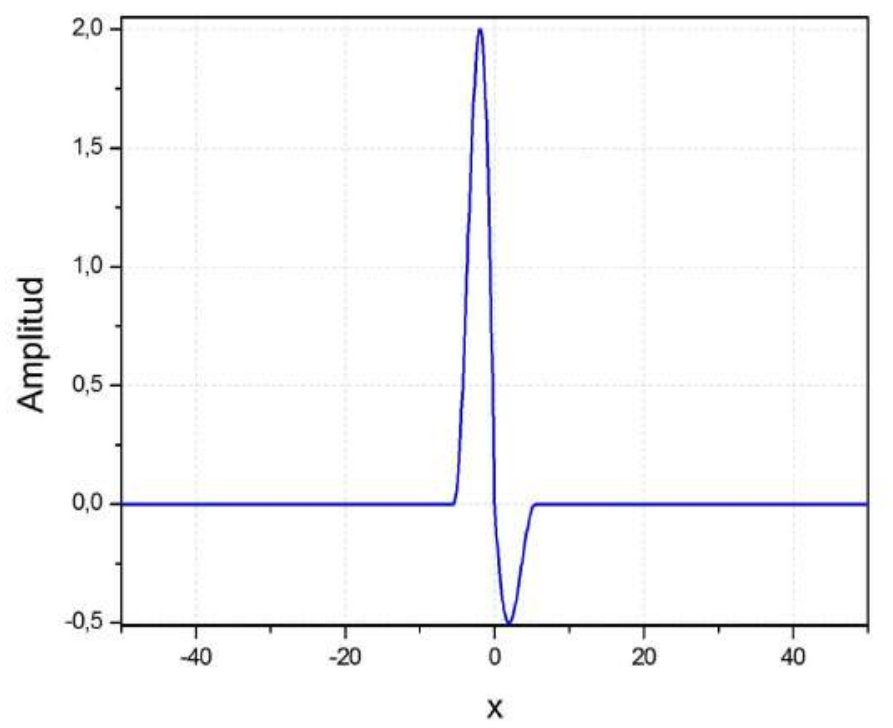

(a)

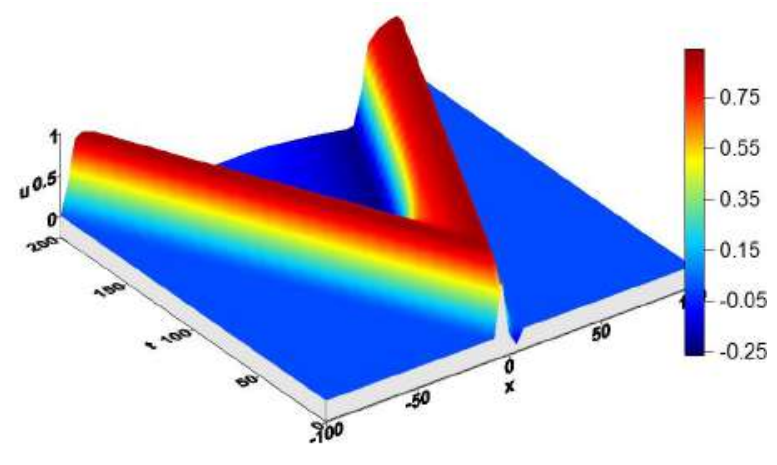

(b)

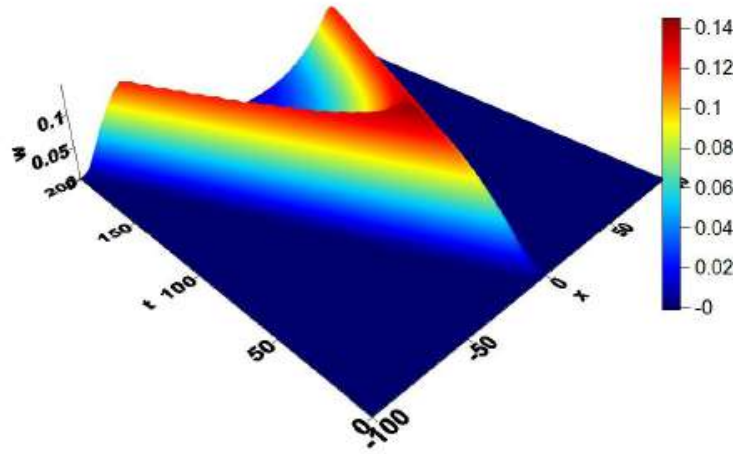

(c)

Figura 4.8. CI de forma senoidal de amplitud 2 (a), propagación de las componentes u en (b) y $w$ en (c) con el sistema FHN en coordenadas locales, sistema (2.1).

y $u_{0}(x)=0$ en el resto. Usamos 1600 elementos cuadráticos en el dominio espacial y el paso temporal $\Delta t=0.5$. Como se muestra en la Fig. 4.10, dos pulsos viajeros son obtenidos en cada punto de localización de los pulsos de excitación. Cuando las dos ondas viajeras colisionan, no se observa un efecto típico de interferencia de ondas, las ondas viajeras se aniquilan entre sí, este es un fenómeno propio del modelo FHN, presentes en los medios excitables como el cerebro y el corazón. Este fenómeno de aniquilación es explorado en la eliminación de arritmias que será estudiado enun próximo trabajo.

5. Conclusiones. En este trabajo hemos usado el Software de distribución libre FreeFem++ para resolver el modelo de FHN unidimensional. Presentamos una amplia gama de ejemplos de simulaciones los cuales fueron hechos con el objetivo de entender los fenómenos físicos presentes en medios excitables, bien como también obtener experiencia en el uso de Software antes mencionado.

En trabajos futuros haremos simulaciones de ondas viajeras del tipo espirales, las cuales son generadas en medios excitables como las membranas cerebrales o en los impulsos cardiacos. Los ejemplos de simulación de espirales serán hechos con un sistema FHN bidimensional.

El sistema FHN que hemos implementado es solo un modelo fenomenológico simple para modelar medios excitables que ha sido adaptado para tejidos cardiacos o impulsos eléctricos en membranas nerviosas. Como tal, cualquier afirmación real sobre la aplicabilidad biológica requiere que este mecanismo sea estudiado más rigurosamente. 


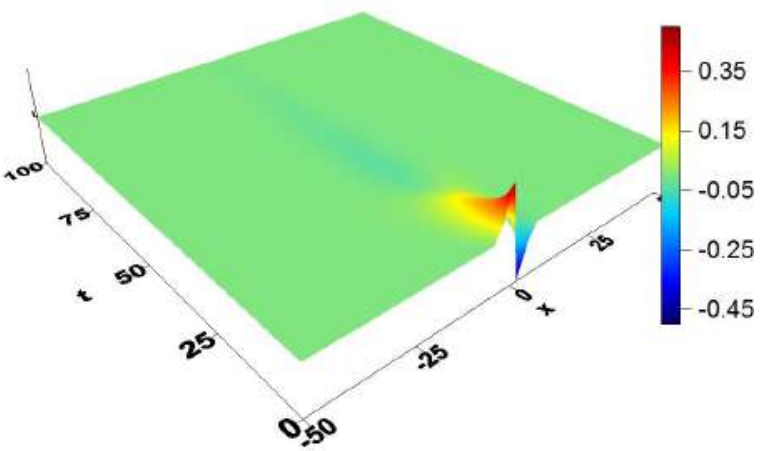

(a)

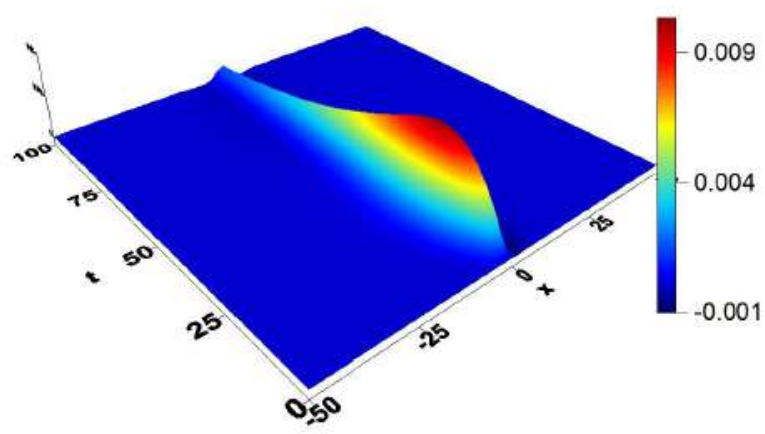

(b)

Figura 4.9. Propagacion de las componentes $u$ (a) y $w$ (b) con el sistema en coordenadas locales (2.1) y para una CI de forma senoidal cuando la amplitud es igual a 0.5 .

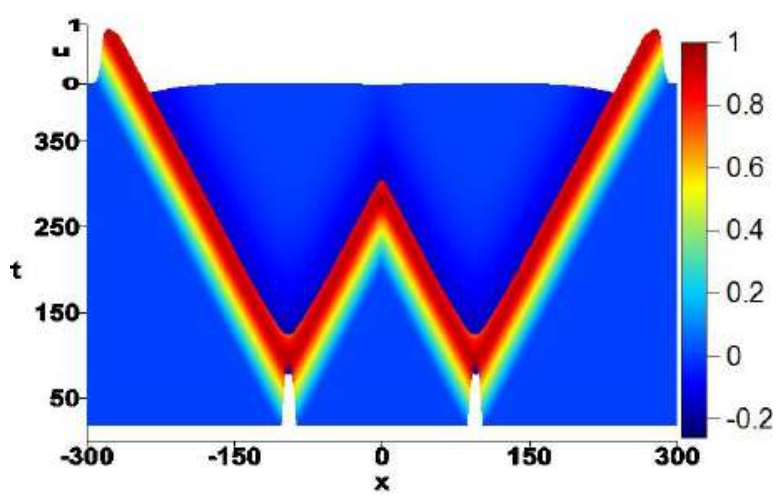

(a)

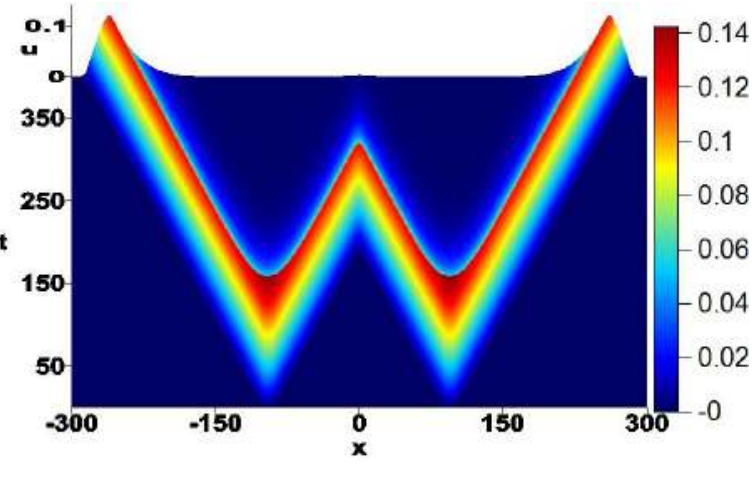

(b)

Figura 4.10. Propagación de las componentes $u(a)$ y $w$ (b) para la simulación de múltiplos pulsos con el sistema en coordenadas locales (2.1). Las ondas se aniquilan cuando colisionan entre sí.

\section{Referencias}

[1] Fitzhugh, R., Impulses and physiological states in theoretical models of nerve membrane, Biophysical Journal, 1 (1961) $445-466$.

[2] Glass, L. Time series analysis of complex dynamics in phisiology and medicine, Natural Sciences and Engineering Research Council of Canada (1992).

[3] Nagumo, J.S. et al An active pulse transmission line simulating nerve axon, Proc.IRE, 50 (1962) 2061-2071.

[4] Panfilov, A. V., Pertsov, A.M., Vortex ring in three-dimensional active medium in reaction-diffusion system, Doklady AN SSSR, 274 (1984) 1500-1503.

[5] Gao, W. y Wang, J., Existence of wavefronts and impulses to FitzHugh-Nagumo equations. Nonlinear Analysis: Theory, Methods and Applications, 57(5): 667-676. 2004.

[6] Yanagida, E., Stability of travelling front solutions of the FitzHugh-Nagumo equations. Mathematical and Computer Modelling, 12(3): 289-301. 1989.

7] Hecht, F., New development in FreeFem++, J. Numer. Math. 20(2012), no. 3-4, 251-265.

[8] FreeFem++ Free Manual. http://www.freefem.org. [accessed 10-December-2018].

[9] Arrieta, J. M. Lopez-Fernandez, M. y Zuazua, E., Approximating travelling waves by equilibria of non local equations. Asymptotic Analysis, (78): 145-186. 2012.

[10] Beyn, W., Otter, D. y Rottmann-Matthes, J., Stability and computation of dynamic patterns in PDEs, Current Challenges in Stability Issues for Numerical DE, 89-172, 2011.

[11] Feng, H., Numerical simulation of the FHN model with strong reaction, Master thesis, Texas A and M International University, 2012.

[12] Paton, K. M., A study of wave propagation in the FHN system, Master thesis, University of British Columbia, 2011.

[13] Verão, G. B., , Aproximando ondas viajantes por equilíbrio de uma equação não local, Tese de doutorado, USP, 2016. 